\title{
Antigen specificity of antihistone antibodies in systemic sclerosis
}

\author{
Minoru Hasegawa, Shinichi Sato, Kanako Kikuchi, Kazuhiko Takehara
}

\begin{abstract}
Objectives-The aim of the study was to determine the prevalence and clinical significance of antibodies to individual histone components in systemic sclerosis (SSc).

Methods-Serum samples from patients with limited cutaneous SSc $(1 S S c ; n=42)$ and diffuse cutaneous SSc (dSSc; $n=28)$ were examined for IgG and/or IgM antibodies to individual histone components and complexes by enzyme linked immunosorbent assay (ELISA).

Results-The level of IgG antibody to total histones was significantly higher in ISSc and dSSc than in normal controls. The level of IgM antibody to total histones was significantly higher in 1SSc, but not in dSSc, than in normal controls. IgG antibody to total histones tended to be increased in dSSc when compared with that in 1SSc. On the other hand, IgM antibody to total histones tended to be increased in 1SSc when compared with that in dSSc. Although SSc showed various antihistone specificities, H2B, H2A-H2B, (H2A-H2B)dsDNA were main antigens recognised by IgG antibodies in both $1 S S c$ and dSSc. Although IgM antibodies to $\mathrm{H} 2 \mathrm{~B}$ and $\mathrm{H} 2 \mathrm{~A}$ H2B were also detected in both 1SSc and dSSc, serum samples from 1SSc patients exhibited highest IgM reactivity with $H 1$. Conclusion-SSc may be included among conditions in which heterogeneous antihistone antibodies are produced. IgM antibodies to the most accessible histone H1 may be related to mild clinical features (ISSc) and IgG antibodies to the inner core molecules of native histone such as H2B or complexes including $\mathrm{H} 2 \mathrm{~B}$ may be associated with severe clinical features (dSSc) in Ssc.

(Ann Rheum Dis 1998;57:470-475)
\end{abstract}

Antihistone antibodies (AHAs) are commonly observed in drug induced lupus erythematosus (drug induced LE) and systemic lupus erythematosus (SLE) ${ }^{1}$ However, AHAs have also been detected in patients with rheumatoid arthritis (RA), systemic sclerosis (SSc), localised scleroderma, and other diseases related to the presence of autoantibodies. ${ }^{2-4}$

In addition, antibodies to individual histone components and complexes have also been investigated in some diseases with AHAs. Among them, antibodies to the H2A-H2B complex and (H2A-H2B)-DNA complex have been reported to be a more sensitive and specific marker for drug induced LE than those to individual histone classes. ${ }^{5}$

Previously, we detected AHAs in $29 \%$ of patients with SSc by means of enzyme linked immunosorbent assay (ELISA) and indicated that the presence of AHAs was correlated with the presence of severe pulmonary fibrosis in these patients. ${ }^{3}$ Subsequently, another group reported that the patients with AHAs had cardiac and renal involvement more frequently than the patients without AHAs. ${ }^{6}$

However, the exact antigen specificity of AHAs in SSc is still unknown. In this study, we determined the level of antibodies against individual histone components and complexes in serum samples from patients with SSc to clarify the antigen specificity to AHAs. Furthermore, we also determined the relation between these antibodies and the clinical features. Our data suggest that IgM antibodies to the most accessible histone $\mathrm{H} 1$ may be related to mild clinical features (1SSc) and IgG antibodies to the inner core molecules of native histone such as $\mathrm{H} 2 \mathrm{~B}$ or complexes including $\mathrm{H} 2 \mathrm{~B}$ may be associated with severe clinical features $(\mathrm{dSSc})$ in patients with SSc.

\section{Methods}

PATIENTS AND CONTROLS

Table 1 shows the demographic features of patients with SSc and controls included in the

K Takehara

Department of Dermatology, Faculty of Medicine,

University of Tokyo, Tokyo, Japan

K Kikuchi

Correspondence to Dr S Sato, Department of Dermatology, Kanazawa University School of Medicine, 13-1,

Takara-machi, Kanazawa 920-8641, Japan.

Table 1 Characteristics of patients with systemic sclerosis (SSc) and controls included in the study

\begin{tabular}{|c|c|c|c|c|c|c|c|c|c|}
\hline \multirow[b]{2}{*}{ Group } & \multirow[b]{2}{*}{ Number } & \multirow{2}{*}{$\begin{array}{l}\text { Sex } \\
\text { (male/female) }\end{array}$} & \multirow{2}{*}{$\begin{array}{l}\text { Age }(y \\
(S D))\end{array}$} & \multirow{2}{*}{$\begin{array}{l}\text { Disease } \\
\text { duration } \\
(y(S D))\end{array}$} & \multicolumn{3}{|c|}{ Autoantibody (\%) } & \multicolumn{2}{|l|}{ Treatment (\%) } \\
\hline & & & & & Topo-1 & $A C A$ & U1 RNP & Corticosteroid & D-penicillamine \\
\hline All SSc & 70 & $8 / 62$ & $49(15)$ & $8.0(8.4)$ & 37 & 31 & 14 & 33 & 14 \\
\hline $1 \mathrm{SSc}$ & 42 & $1 / 41$ & $50(13)$ & $9.6(9.3)$ & 10 & 48 & 17 & 12 & 5 \\
\hline $\mathrm{dSSc}$ & 28 & $7 / 21$ & $44(18)$ & $5.8(6.4)$ & 79 & 7 & 11 & 64 & 29 \\
\hline SLE & 22 & $4 / 18$ & $34(13)$ & $6.7(5.6)$ & 0 & 0 & 0 & 91 & 0 \\
\hline BP & 20 & $6 / 14$ & $71(12)$ & $3.8(4.2)$ & 0 & 0 & 0 & 80 & 0 \\
\hline Normal & 57 & $6 / 51$ & $48(18)$ & & 0 & 0 & 0 & 0 & 0 \\
\hline
\end{tabular}

Topo-1, anti-topoisomerase I antibody; ACA, anticentromere antibody; U1 RNP, anti-U1 RNP antibody; 1SSc, limited cutaneous SSc; dSSc, diffuse cutaneous SSc; SLE, systemic lupus erythematosus; BP, bullous pemphigoid; Normal, normal controls. 
study. Serum samples were collected from 70 Japanese SSc patients (62 women and 8 men). These patients were between 10-72 years old (mean age 49). All patients fulfilled the criteria proposed by the American College of Rheumatology (formerly, the American Rheumatism Association). ${ }^{7}$ The patients with SSc were grouped according to the classification system proposed by LeRoy et $a l^{8}$ : 42 patients (41 women and $1 \mathrm{man}$ ) had limited cutaneous SSc (ISSc) and 28 patients (21 women and 7 men) had diffuse cutaneous SSc (dSSc). Normal control serum samples were obtained from 57 healthy Japanese volunteers ( 51 women and 6 men). The age of the normal controls was 15-71 years old (mean age 48). For comparison, serum samples were also obtained from 22 Japanese patients with SLE (19-54 years old, mean age 34) and 20 Japanese patients with bullous pemphigoid (BP), an autoimmune skin disease in which AHAs do not occur (48-88 years old, mean age 71).

Fresh venous blood samples were centrifuged shortly after clot formation. All samples were stored at $-70^{\circ} \mathrm{C}$ before use. The clinical and laboratory data reported herein were obtained at the time the serum samples were drawn. Patients had a detailed clinical assessment and the involvement of their organ systems was investigated. Organ system involvement was defined as described by Steen et $a l,{ }^{9}$ with some modifications: lung=bibasilar fibrosis on chest radiography; oesophagus =hypomotility shown by barium radiography, joint=inflammatory polyarthralgias or arthritis, muscle=proximal muscle weakness and increased serum creatine kinase. Erythrocyte sedimentation rates (ESR), $C$ reactive protein (CRP), IgG, IgA, IgM were considered to be raised when each value was higher than $20 \mathrm{~mm}$ $1 \mathrm{st} \mathrm{h}, 0.5 \mathrm{mg} / \mathrm{dl}, 1774 \mathrm{mg} / \mathrm{dl}, 235 \mathrm{mg} / \mathrm{dl}, 355$ $\mathrm{mg} / \mathrm{dl}$, respectively. Antinuclear antibody was detected by indirect immunofluorescence, using HEp-2 cells as the substrate, and by double immunodiffusion.

ELISA FOR ANTIBODIES TO HISTONES

Calf thymus total histones (Sigma, St Louis, $\mathrm{MO}$ ) and histone $\mathrm{H} 1, \mathrm{H} 2 \mathrm{~A}, \mathrm{H} 2 \mathrm{~B}, \mathrm{H} 3$, and $\mathrm{H} 4$ (Boehringer Mannheim, Mannheim, Germany) were purchased, and double strand DNA (dsDNA) was obtained from the Medical and Biological Laboratories (Nagoya, Japan). $\mathrm{H} 2 \mathrm{~A}-\mathrm{H} 2 \mathrm{~B}$ complexes and (H2A-H2B) -dsDNA complexes were prepared as described previously. ${ }^{10}$ Antibodies to these histone components were determined by ELISA as described elsewhere, with some modifications. ${ }^{11}$ Briefly, microtitre assay plates were coated with these histone components at $2.5 \mu \mathrm{g} / \mathrm{ml}$, and serum samples (diluted 1:100) were added. This dilution was in the linear portion of the curve comparing dilution and optical density (OD) (data not shown). Every serum sample was tested in duplicate. The bound antibodies were detected with peroxidase conjugated antihuman IgG or IgM antibodies. The OD of the samples was read at $405 \mathrm{~nm}$ on a microplate reader at 60 minutes after addition of the substrate. If this reading was greater than the sensitivity of the microplate reader (2.0 OD), the final OD was calculated by extrapolation from an earlier reading as described. ${ }^{12}$ That is, the mean ratio of the second and first readings of the three highest samples still under the sensitivity of the microplate reader at the 60 minute time point was determined. The first reading value of samples over detection limits at 60 minutes was multiplied by this factor, yielding the final extrapolated OD. Extrapolation was linear to at least $12 \mathrm{OD}$, thereby expanding the dynamic range of assay. We used some positive control serum samples from patients with SLE to correct for inter-assay or inter-plate variation.

\section{STATISTICAL ANALYSIS}

Initial analysis of variance between groups was performed using Kruskal-Wallis test. Individual between group comparisons were tested by Mann-Whitney U test where indicated. Percentages were compared by $\chi^{2}$ test or Fisher's exact probability test. $p$ Values of less than 0.01 were considered statistically significant.

\section{Results}

ANTIBODIES TO TOTAL HISTONES IN SSC

Figure 1 shows the concentrations of antibodies to total histones present in serum samples studied. OD more than $3 \mathrm{SD}$ above the mean value in normal controls were considered positive in each assay. IgG antibodies to total histones were present in 10 of $70(14 \%)$ patients with SSc and 8 of $22(36 \%)$ patients with SLE. IgM antibodies to total histones were detected in 15 of $70(21 \%)$ patients with SSc and 8 of $22(36 \%)$ patients with SLE. Nineteen of $70(27 \%)$ patients with SSc were positive for IgG and/or IgM antibodies to total histones. Neither IgG nor IgM AHAs were present in the serum samples obtained from patients with BP and normal controls.

IgG antihistone activity in SSc (median 0.512 , range $0-3.128$ ) was significantly higher than those in BP (median 0.237, range $0-0.580, \mathrm{p}<0.005)$ and normal control (median 0.344 , range $0-0.912, \mathrm{p}<0.005$ ), although IgG antihistone activity in SLE (median 0.820, range 0-5.200) was significantly higher than that in SSc $(p<0.01)$. Concerning the subgroups of SSc, IgG antihistone activity in dSSc (median 0.536, range 0-3.128) was significantly higher than those in BP and normal controls. IgG antihistone activity in $1 \mathrm{SSc}$ (median 0.512, range 0-1.752) was also significantly higher than those in BP and normal controls. Although IgG antihistone activity in dSSc was higher than that in ISSc, it was not significant. IgG antihistone activity in SLE was significantly higher than those in 1SSc, BP, and normal controls.

IgM antihistone activity in SSc (median 0.620 , range $0-5.400$ ) was significantly increased when compared with those in BP (median 0.292, range $0-0.730, \mathrm{p}<0.005$ ) and normal controls (median 0.312, range $0-1.200, \mathrm{p}<0.01)$. Although $\operatorname{IgM}$ antihistone activity in SLE (median 1.164, range 0.1124.512) was higher than that in SSc, it was not significant. IgM antihistone activity in $1 \mathrm{SSc}$ 
(median 0.708, range 0-5.300) was significantly increased when compared with those in $\mathrm{BP}$ and normal controls. Although $\operatorname{IgM}$ antihistone activity in dSSc (median 0.388,
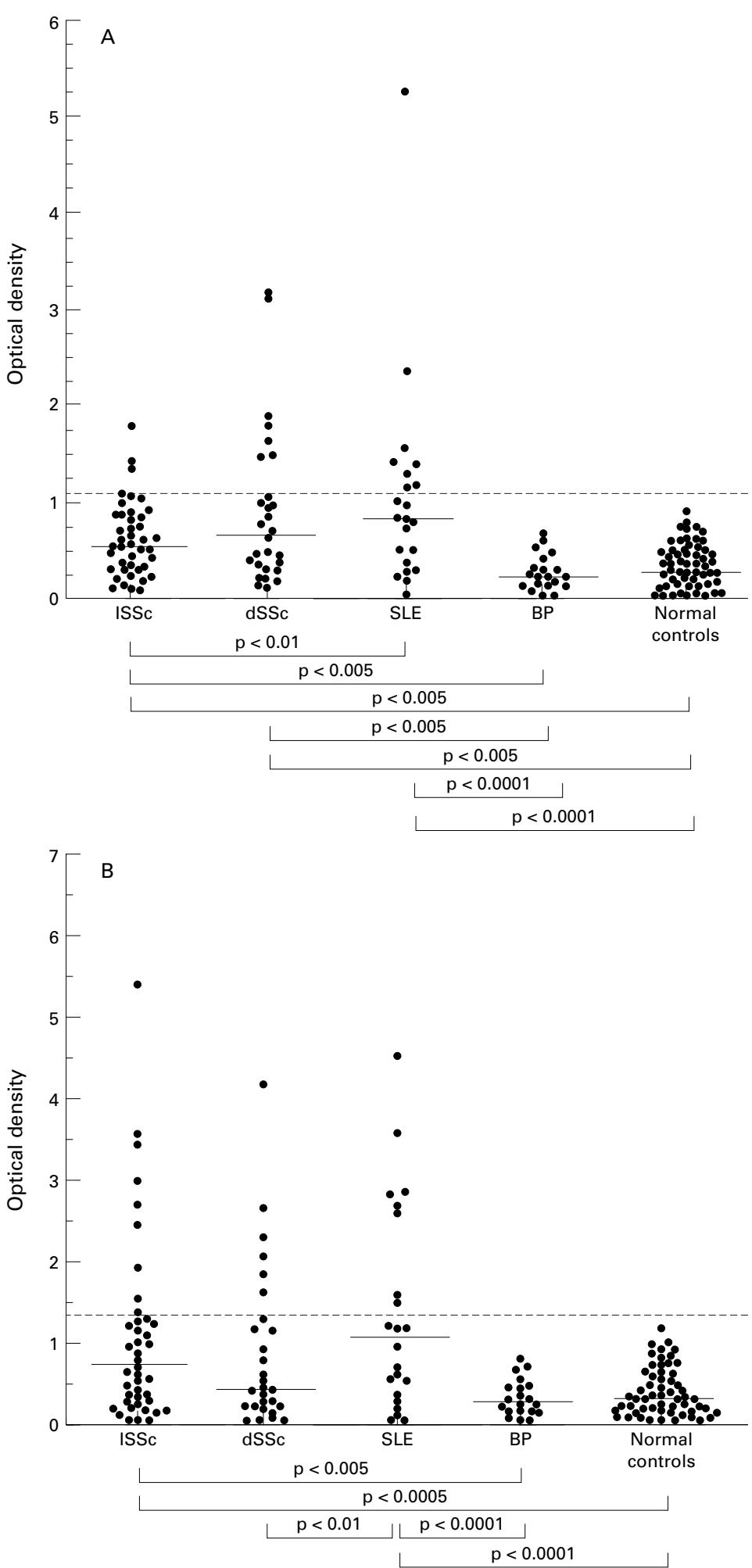

FIgure 1 ELISA for antibody to total histones in serum samples from patients with limited cutaneous systemic sclerosis (ASSc) (n=42), diffuse cutaneous systemic sclerosis $(d S S c)(n=28)$, systemic lupus erythematosus (SLE) ( $n=22)$, bullous pemphigoid (BP) $(n=20)$, and normal controls $(n=57)$. Horizontal broken line represents the median value $+3 S D$ of antihistone activity in normal control group. Horizontal short bars represent the median values in each group. (A) Serum IgG reactivity to total histones in $I S S c$, dSSc, $S L E, B P$, and normal control. (B) Serum IgM reactivity to total histones in ISSc, dSSc, $S L E, B P$, and normal control. range 0-4.200) was also increased when compared with those in BP and normal controls, they were not significant. IgM antihistone activity in SLE was significantly increased than those in dSSc, BP, and normal controls.

Thus, we confirm the previous reports in which AHAs were detected in serum samples from SSc, although at lower frequency and concentration than SLE. In addition, we found that the level of IgG antihistone activity tended to be increased in dSSc when compared with that in ISSc, and that the level of IgM antihistone activity tended to be increased in 1SSc when compared with that in dSSc.

LEVELS OF ANTIBODIES TO EACH HISTONE COMPONENT AND COMPLEX IN SSC

The reactivity with each antigen of normal control was determined, and this average OD was subtracted from the reactivity of each patient's serum on that antigen for comparing the average binding to each histone component and complex.

The average OD of IgG reactivity was highest for H2B, followed by (H2A-H2B)-dsDNA, $\mathrm{H} 2 \mathrm{~A}-\mathrm{H} 2 \mathrm{~B}, \mathrm{H} 3, \mathrm{H} 2 \mathrm{~A}, \mathrm{H} 1$ in patients with SSc (fig $2 \mathrm{~A}$ ). The pattern of reactivity of IgG antibodies with individual histones and complexes in 1SSc was similar to that in dSSc, although the reactivity tended to be higher in $\mathrm{dSSc}$ when compared with those in 1SSc (fig $2 \mathrm{~B}, \mathrm{C}$ ). The main antigens recognised by IgG AHAs in both $1 \mathrm{SSc}$ and $\mathrm{dSSc}$ were $\mathrm{H} 2 \mathrm{~B}, \mathrm{H} 2 \mathrm{~A}-\mathrm{H} 2 \mathrm{~B}$, and (H2A-H2B)-dsDNA.

$\mathrm{IgM}$ reactivity was highest with $\mathrm{H} 2 \mathrm{~A}-\mathrm{H} 2 \mathrm{~B}$, followed by $\mathrm{H} 1, \mathrm{H} 2 \mathrm{~B}$, (H2A-H2B)-dsDNA, $\mathrm{H} 2 \mathrm{~A}$ in patients with SSc (fig 3A). Although IgM antibodies against $\mathrm{H} 2 \mathrm{~A}-\mathrm{H} 2 \mathrm{~B}, \mathrm{H} 2 \mathrm{~B}$, and (H2A-H2B)-dsDNA were increased in $1 \mathrm{SSc}$, the serum samples from patients with $1 S S c$ exhibited highest IgM reactivity with $\mathrm{H} 1$ (fig 3B). This shows that $\mathrm{H} 1$ may be a main antigen recognised by IgM-AHAs in $1 S S c$. In contrast, IgM reactivity with $\mathrm{H} 2 \mathrm{~A}-\mathrm{H} 2 \mathrm{~B}$ was highest among individual histone components and complexes in dSSc (fig 3C), although at lower levels than that in 1SSc. There was a distinct lack of IgM reactivity with $\mathrm{H} 3$ and $\mathrm{H} 4$ in patients with SSc.

CORRELATIONS BETWEEN AUTOANTIBODIES AND THE CLINICAL FEATURES

The presence of IgG antibodies, but not $\operatorname{IgM}$ antibodies, to total histones showed a tendency to correlate with the presence of pulmonary fibrosis in patients with SSc. That is, the frequency of pulmonary fibrosis in SSc patients with IgG antibodies to total histones was higher than in SSc patients without IgG antibodies to total histones ( $60 \%$ versus $20 \%$, $\mathrm{p}=0.01)$. IgG antibodies to total histones were detected in $0 \%, 20 \%, 18 \%$ of patients with anticentomere antibody (ACA), anti-U1 RNP antibody, and anti-topoisomerase I antibody, respectively. ACA was detected less frequently in SSc patients with IgG antibodies to total histones than in SSc patients without IgG antibodies to total histones ( $0 \%$ versus $37 \%$, $\mathrm{p}=0.02)$. IgM antibodies to total histones were detected in $18 \%, 31 \%, 30 \%$ of patients with 

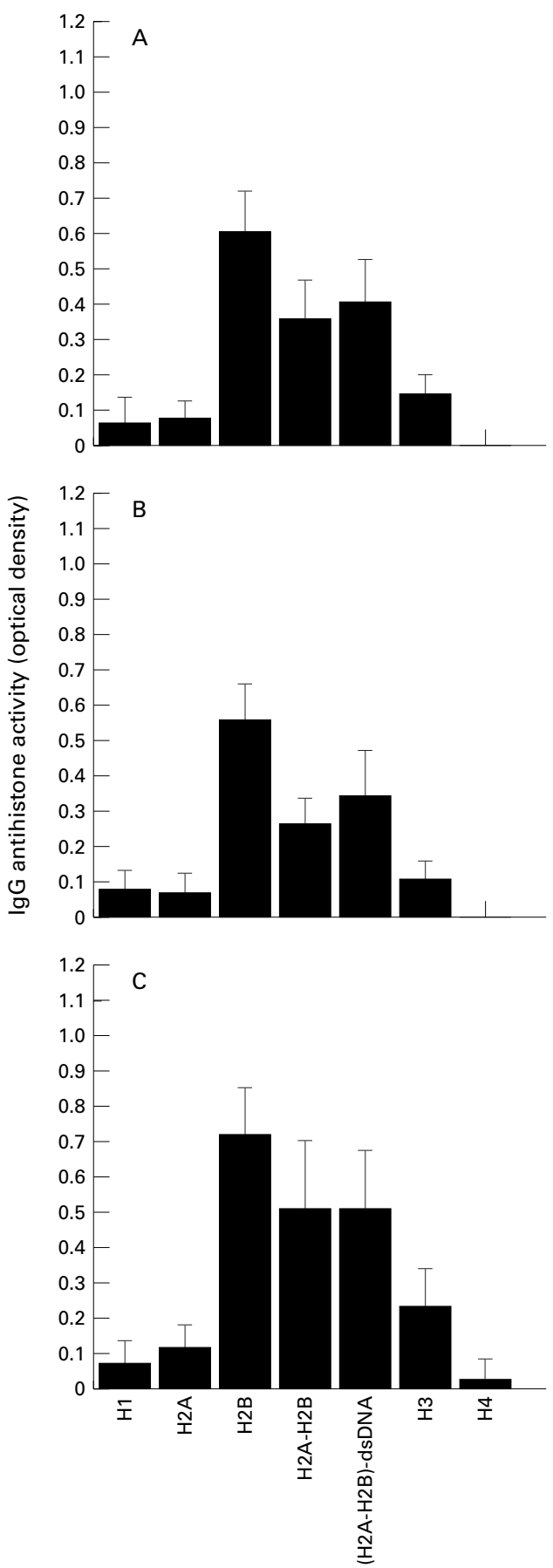

Figure 2 Patterns of $\operatorname{Ig} G$ reactivity of antihistone antibodies with individual histone components and complexes in patients with systemic sclerosis (SSc). Average $O D$ of IgG reactivity to each histone in normal controls was substracted from the $O D$ (mean (SEM)) of IgG reactivity to each histone in patients. (A) IgG reactivity to histones in patients with $S S c(n=70),(B) \operatorname{IgG}$ reactivity to histones in patients with limited cutaneous SSc (ISSc) $(n=42),(C)$ IgG reactivity to histones in patients with diffuse cutaneous $S S c(d S S c)(n=28)$.

ACA, anti-U1 RNP antibody, and antitopoisomerase I antibody, respectively. Otherwise, no correlations were found between the presence of IgG or IgM antibodies to total histones and the clinical or laboratory features. Furthermore, we determined the association of antibodies to each of the seven individual histone components and complexes with the clinical and laboratory features. However, as a
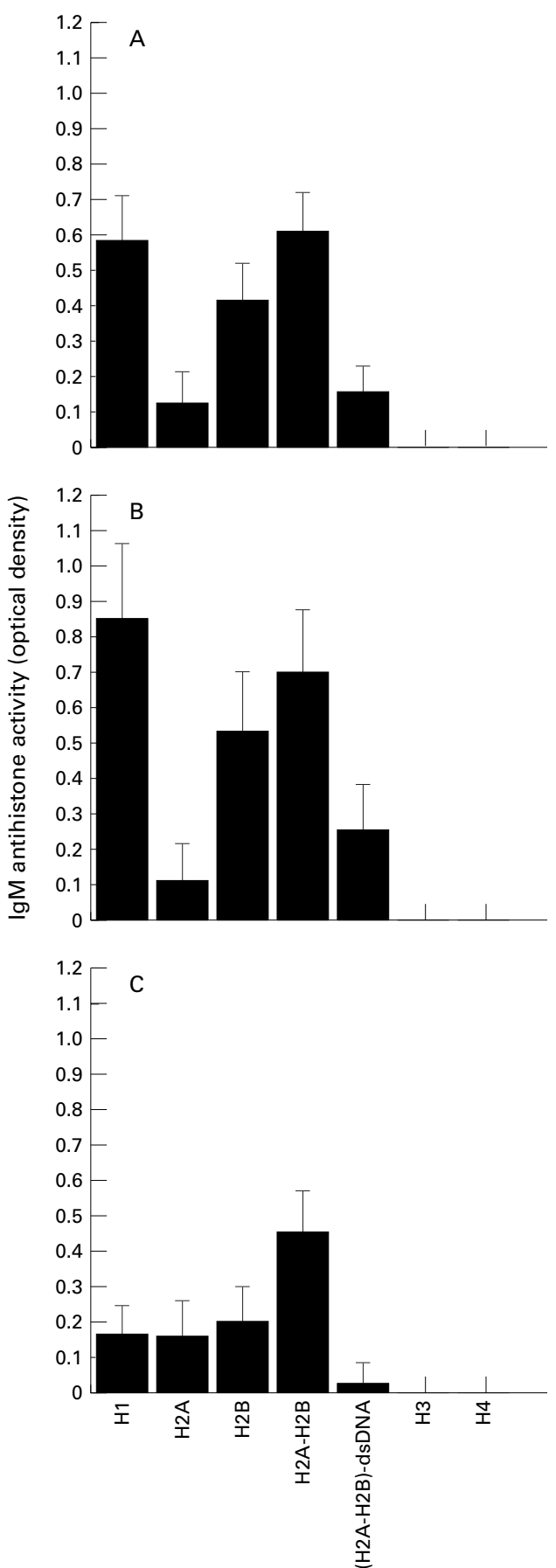

Figure 3 Patterns of IgM reactivity of antihistone antibodies with individual histone components and complexes in patients with systemic sclerosis (SSc). Average OD of IgM reactivity to each histone in normal controls was substracted from the OD (mean (SEM)) of IgM reactivity to each histone in patients. (A) IgM reactivity to histones in patients with $S S c(n=70)$, (B) IgM reactivity to histones in patients with limited cutaneous SSc (ISSc) $(n=42),(C)$ IgM reactivity to histones in patients with diffuse cutaneous $S S c(d S S c)(n=28)$.

wide diversity of specificities occur within the patient population, no correlations were observed between each antibody and the presence of any clinical features or laboratory abnormalities (data not shown). There were no correlations between the presence of AHAs and the duration of SSc. Levels of AHAs in patients who were being treated with prednisolone (2.5-20 mg/day) or D-penicillamine (100- 
$300 \mathrm{mg} /$ day) were not significantly different from those not taking them (data not shown).

\section{Discussion}

In this study, we showed that the antihistone responses in SSc are heterogeneous among patients with SSc. However, some findings are observed between antihistone antibodies and the clinical features. Concerning the isotype of AHAs, IgG reactivities to total histones tended to be increased in patients with dSSc, and IgM reactivities to total histones tended to be increased in patients with $1 \mathrm{SSc}$. IgG-AHAs to total histones showed a tendency to be detected frequently in patients with pulmonary fibrosis and in patients without ACA. In addition, we demonstrated that $\mathrm{H} 2 \mathrm{~B}$ or complexes including $\mathrm{H} 2 \mathrm{~B}$ are predominant antigens recognised by IgG antibodies in both $1 S S c$ and dSSc. Although H2B or complex including $\mathrm{H} 2 \mathrm{~B}$ are also recognised by $\operatorname{IgM}$ antibodies in both $1 \mathrm{SSc}$ and $\mathrm{dSSc}, \mathrm{H} 1$ is a main antigen recognised by IgM antibodies in 1SSc. Therefore, these findings suggest that IgM antibody to $\mathrm{H} 1$ may be a main antibody among AHAs detected in serum samples from patients with $1 S S c$, and that IgG antibodies to $\mathrm{H} 2 \mathrm{~B}$ or complex including $\mathrm{H} 2 \mathrm{~B}$ in absence of antibodies against $\mathrm{H} 1$ may be a characteristic of AHAs seen in serum samples from patients with dSSc.

Previously, we demonstrated the significant correlation between antibodies to total histones and pulmonary fibrosis. ${ }^{3}$ In this study, we confirm that the presence of IgG antibodies, but not IgM antibodies, to total histones tends to correlate with the pulmonary fibrosis in different SSc patients group. Furthermore, we found that IgG antibodies to total histones were especially increased in patients without ACA. It is well known that the presence of pulmonary fibrosis reduces survival in SSc. In addition, patients with ACA generally do not show severe clinical features. Therefore, our findings suggest that the presence of IgG antibodies to total histones is associated with severe clinical features in SSc.

Our previous study also demonstrated that IgG antibodies to total histones in SSc specifically reacted with $\mathrm{H} 1$ by immunoblotting. ${ }^{3}$ However, in this study, AHAs reacted with various types of histone components in patients with SSc. This discrepancy may be because of differences in the detecting assays, because we reported difference in reactivities to each histone component between immunoblotting and ELISA in patients with localised scleroderma, which was similar to the difference observed in this study. ${ }^{13}$ Samples processed for immunoblotting procedures are boiled in a sodium dodecyl sulphate solution to deliberately unfold a higher ordered structure. A previous study has shown that serum samples with antibodies to the H2A-H2B complex from procainamide induced lupus patients detected a sixfold to 10 -fold reduction in their ability to react with $\mathrm{H} 2 \mathrm{~A}-\mathrm{H} 2 \mathrm{~B}$ complexes treated with sodium dodecyl sulphate. ${ }^{14}{ }^{15}$ Thus, the irreversible denaturation of conformational antigenic determinants may tend to be selective for epitopes generated only by linear amino acid sequences. This substrate denaturation process is not required by ELISA, and the antigen seems to maintain a more native protein structure than in immunoblotting.

In our experiments, antibodies to (H2AH2B)-dsDNA were not necessarily specific for SSc when compared with antibodies to the other histone components. In a previous study with a small number of patients, it was reported that antibodies to (H2A-H2B)-dsDNA in the absence of antibodies to dsDNA or to $\mathrm{H} 2 \mathrm{~A}$ $\mathrm{H} 2 \mathrm{~B}$ are more sensitive and specific for scleroderma related disorders than for SLE. ${ }^{16}$ The discrepancy between our study and the previous study may be in part attributed to differences in the patient groups studied and antigens used for the ELISA. Their patient group included not only patients with SSc but also patients with mixed connective tissue disease and localised scleroderma (morphea). Although they measured antibodies to dsDNA, $\mathrm{H} 2 \mathrm{~A}-\mathrm{H} 2 \mathrm{~B}$, and (H2A-H2B)-dsDNA, antibodies to other histone components were not investigated. Our results demonstrate that patients with SSc have a variable antihistone profiles in a larger number of patients.

Our results suggest that the antibodies to histones are directed against the native chromatin itself in SSc, as they reacted with the relatively exposed portion of chromatin. Previous biochemical studies show that each nucleosome is composed of an inner core of two molecules each of $\mathrm{H} 3$ and $\mathrm{H} 4$ and the outer portion of two molecules of $\mathrm{H} 2 \mathrm{~A}$ and $\mathrm{H} 2 \mathrm{~B}$. In the complete nucleosome, $\mathrm{H} 1$ occupies a particularly exposed external position, and its antigenic $\mathrm{COOH}$ terminal tail is binding linker DNA. The $\mathrm{NH}_{2}$ terminal half of $\mathrm{H} 2 \mathrm{~B}$ is also in a relative exposed position within the nucleosome. Thus, most investigators agree that in native chromatin the epitopes of $\mathrm{H} 1$ are most accessible, followed by the epitopes of $\mathrm{H} 2 \mathrm{~B}$, $\mathrm{H} 3, \mathrm{H} 2 \mathrm{~A}$, and $\mathrm{H} 4$. $^{17-19}$ The autoimmune response is considered to be antigen driven and to be directed towards various components of the subcellular particle, consisting of different species of proteins or of proteins in association with nucleic acids. ${ }^{20}{ }^{21}$ Therefore, our study suggests that AHAs are induced by native chromatin particles as immunogens in SSc, because $\mathrm{H} 1, \mathrm{H} 2 \mathrm{~B}$, or complexes including $\mathrm{H} 2 \mathrm{~B}$, which are mainly recognised by AHAs in $\mathrm{SSc}$, have a relatively strong antigenic nature.

$\operatorname{IgM}$ reactivity with $\mathrm{H} 1$ was significantly increased in patients with $1 S S c$ when compared with dSSc, although $\operatorname{IgM}$ reactivities with $\mathrm{H} 2 \mathrm{~B}, \mathrm{H} 2 \mathrm{~A}-\mathrm{H} 2 \mathrm{~B}$, (H2A-H2B)-dsDNA were also detected in 1SSc. Therefore this finding suggests that antihistone antibody to the most accessible histone (H1) may be a serological indicator for mild clinical features (1SSc). In contrast, the inner core molecules of native histones, including $\mathrm{H} 2 \mathrm{~B}, \mathrm{H} 2 \mathrm{~A}-\mathrm{H} 2 \mathrm{~B}$, ( $\mathrm{H} 2 \mathrm{~A}-$ $\mathrm{H} 2 \mathrm{~B}$ )-dsDNA, H3, H4, were predominant antigens recognised by the serum samples from patients with dSSc. Furthermore, antibodies to these antigens were mainly IgG in dSSc. Therefore, IgG antibodies to $\mathrm{H} 2 \mathrm{~B}, \mathrm{H} 2 \mathrm{~A}-\mathrm{H} 2 \mathrm{~B}$, and (H2A-H2B)-dsDNA in absence of antibodies to $\mathrm{H} 1$ might be a serological indicator 
for more severe clinical features (dSSc). The presence of IgG antibodies against $\mathrm{H} 2 \mathrm{~B}, \mathrm{H} 2 \mathrm{~A}$ $\mathrm{H} 2 \mathrm{~B}$, and (H2A-H2B)-dsDNA in dSSc could be explained by spreading of the immune response to the inner portion of chromatin.

This study was supported by a research grant from the Scleroderma Research Committee of the Ministry of Health and Welfare, Japan.

1 Fritzler MJ, Tan EM. Antibodies to histones in druginduced and idiopathic lupus erythematosus. J Clin Invest 1978;62:560-7.

2 Aitcheson CT, Peebles C, Joslin F, Tan EM. Characteristics of antinuclear antibodies in rheumatoid arthritis. Arthritis Rheum 1980;23:528-38.

3 Sato S, Ihn H, Kikuchi K, Takehara K. Antihistone antibodies in systemic sclerosis: association with pulmonary fibroies in systemic sclerosis: association
sis. Arthritis Rheum 1994;37:391-4.

4 Sato S, Ihn H, Soma Y, Igarashi A, Tamaki T, Kikuchi K, et al. Antihistone antibodies in patients with localized scleroderma. Arthritis Rheum 1993;36:1137-41.

5 Burlingame RW, Rubin RL. Drug-induced anti-histone autoantibodies display two patterns of reactivity with substructures of chromatin. J Clin Invest 1991;88:680-90

6 Parodi A, Drosera M, Barbieri L, Rebora A. Antihistone antibodies in scleroderma. Dermatology 1995;191:16-18.

7 Subcommittee for Scleroderma Criteria of the American Rheumatism Association Diagnostic and Therapeutic Criteria Committee. Preliminary criteria for the classification of systemic sclerosis (scleroderma). Arthritis Rheum 1980; 23:581-90.

8 LeRoy EC, Black C, Fleischmajer R, Jablonska S, Krieg T, Medsger TA, et al. Scleroderma (systemic sclerosis): classification, subsets and pathogenesis. J Rheumatol 1988;15: $202-5$.

9 Steen VD, Powell DL, Medsger TA Jr. Clinical correlations and prognosis based on serum autoantibodies in patients with systemic sclerosis. Arthritis Rheum 1988;31:196-203.
10 Rubin RL, Bell SA, Burlingame RW. Autoantibodies associated with lupus induced by diverse drugs target a similar histone in the (H2A-H2B) -DNA complex. J Clin Invest 1992;90:165-73.

11 Rubin RL. Enzyme-linked immunosorbent assay for antiDNA and antihistone antibodies including anti-(H2AH2B). In: Rose NR, Friedman H, Fahey JL, eds. Manual of clinical laboratory immunology Washington, DC: American Society for Microbiology, 1986:744-9.

12 Rubin RL. Anti-DNA antibodies. In: Bergmeyer H, ed. Methods of enzymatic analysis. New York: Academic Press, 1986:175-89.

13 Sato S, Fujimoto M, Ihn H, Kikuchi K, Takehara K. Antigen specificity of antihistone antibodies in localized scleroderma. Arch Dermatol 1994;130:1273-7.

14 Rubin RL, Waga S. Antihistone antibodies in systemic lupus erythematosus. J Rheumatol 1987;14 (suppl 13):118-26.

15 Portanova JP, Arndt RE, Tan EM, Kotzin BL. Anti-histone antibodies in idiopathic and drug-induced lupus recognize distinct intrahistone regions. J Immunol 1987;138:446-51.

16 Wallace DJ, Lin HC, Shen GQ, Peter JB. Antibodies to histone (H2A-H2B) -DNA complexes in the absence of antibodies to double-strand DNA or to $(\mathrm{H} 2 \mathrm{~A}-\mathrm{H} 2 \mathrm{~B})$ complexes are more sensitive and specific for scleroderma-related disorders than for lupus. Arthritis Rheum 1994;37:1795-7.

17 Goldblatt D, Bustin M, Sperling R. Heterogeneity in the interaction of chromatin subunits with anti-histone sera visualized by immuno-electron microscopy. Exp Cell Res 1978;112:1-14.

18 Absolom D, Van Regenmortel MHV. Nucleosome structure studied with purified antibodies to histones $\mathrm{H} 2 \mathrm{~B}, \mathrm{H} 3$ and H4. FEBS Lett 1978;85:61-4.

19 Muller S, Soussanieh A, Bouley JP, Reinbolt J, Van Regenmortel MHV. Localization of two antigenic determinants in histone H4. Biochem Biophys Acta 1983;747:100-6.

20 Hardin JA. The lupus autoantigens and the pathogenesis of systemic

21 Tan EM Autoantibodies to nuclear antigens (ANA): their immunobiology and medicine. Adv Immunol 1982;33: $167-240$. 